\title{
Global Uncalibrated Visual Servoing for Constrained Robots Working on an Uncalibrated Environments
}

\author{
E.C Dean-León, V. Parra-Vega, Mechatronics Division, CINVESTAV-IPN \\ Av. IPN \# 2508, San Pedro Zacatenco, México, D.F., 07300 Mexico. Email: edean,vparra@ cinvestav.mx \\ A. Espinosa-Romero, Faculty of Mathematics, Universidad Autónoma de Yucatán \\ Mérida, Yucatán, México. Email: eromero@tunku.uady.mx
}

\begin{abstract}
This paper studies the open problem of uncalibrated visual servoing for constrained robots with parametric uncertainties, interacting with unknown environments. A new theoretical framework and its experimental validation are given. This approach produces global exponential convergence of the constrained visual position and the contact force between the end-effector and the unknown constrain surface. This approach is based on the formulation of the Visual Orthogonalization Principle (VOP), which, under the framework of passivity, yields a closed-loop system that fuses camera, encoder and force sensor signals. An experimental system running on Linux-RTAI OS is implemented to visually drive a constrained direct-drive robot manipulator, equipped with six axis JR3 force sensor and a FireWire CCD digital camera posed in fixed configuration. This experimental system proves the effectiveness of the proposed approach even when the robot, camera and restriction surface parameters are assumed as unknown.
\end{abstract}

\section{INTRODUCTION}

In these present days, robot manipulators must perform high precision tasks in more and more complex environments. The task specifications has changed with time and technology, now robots must perform tracking motions and, at the same time, interact with its environment, being capable to react to changes that take place in it. An interesting solution to this problem is to integrate to the robot manipulator sensors of different nature, which are able to extend the information of the state of the system and provide information of changes on the environment that surrounds it. Sensors such as, joint encoders, force sensors, digital cameras, range finders, etc., present remarkable and different properties among each one. However, it is easy to notice that if some of the sensors mentioned above are used, at least two situations may occur; for one side, the information of the system will be suffice, allowing to achieve more explicit control decisions, but, for the other side, some sensor signals will be redundant, this implies that a suitable strategy of sensor fusion should be implemented with meticulous and detailed analysis of the robot dynamics including the information of the environment. Moreover, in order to present a practical approach a robust control law should be designed taking into account parametric uncertainty.

In this realm, this article focuses on the paradigm: "design a controller that ensures tracking of image-based trajectories of constrained robots in an unknown environment subject to uncertainties of camera and robot parameters". This task is very relevant in many robotic applications because vision system allows to structure the unknown environment in contact tasks.

The above problem of force visual servoing is a very complex task for a robotic application, since force and vision signals present different natures, i.e., contact forces input are orthogonal to joint velocity, this implies in terms of passivity, contradictory sensor signals. Thus, these sort of robotic tasks arouse significant difficulties, and stand as a robot control paradigm that surpasses the traditional schemes in robot control and sensor fusion, then is required new theoretical frameworks. The use of the Visual Orthogonalization Principle $(V O P)$ [1], allows to blend both approaches into a unique and elegant visual position force robot control scheme.

\section{A. Contribution}

In this paper a new global and formal solution with its experimental evaluation is presented. This scheme is based on second order sliding mode adaptive controller driven by uncalibrated constrained image errors, which solves by first time the problem possed above. The underlying reason that allows to obtain this result is that a new orthogonalized imagebased error manifold, based on the VOP is introduced. Thus, similar results to the case of nonvisual-based orthogonalized principle are obtained. The closed-loop system guarantees global exponential tracking of visual position and force trajectories, working with unknown environment and subject to parametric uncertainties. Furthermore, its experimental validation is presented on a two degree-of-freedom direct-drive manipulator, equipped with a high-end force sensor, interacting with a highly rigid and uncalibrated surface. The simplicity of the controller enhances its practical applications since the desired task is designed in image space, i.e., the user defines the desired task right from the image that she or he sees, see Fig. 1, wherein the fixed camera supplies a perspective of the desired task.

\section{B. Organization}

Section II provides a review on the several aspects of visual position-force robot control, while Section III introduces 
the nonlinear differential algebraic equations of the robot dynamics in error coordinates. Section IV presents a wellknown camera-robot model, while Section V proposes the VOP and its extended error implemented in the open loop error equation derived in Section VII. Section VIII presents the control design. The experimental validation is presented in Section IX, and finally conclusions are stated in Section X.

\section{PREVIOUS WORKS}

Even when a variety of image-based visual servoing schemes for free motion robot manipulators, which include the dynamic model in the stability analysis, have been proposed, in visual force control, it is not the case. In this sort of controls, there remains important open problems, essentially because, from the theoretical viewpoint, visual force servoing involves redundant sensors, thus it is not evident how to handle sensor fusion in a complex nonlinear dynamical system. From the experimental viewpoint, there arises a multi-rate system due to the slow latency of the camera, in comparison to the latency of the encoder and force sensors. Therefore, a theoretical constrained visual servoing scheme must be accompanied with its experimental validation. ${ }^{1}$

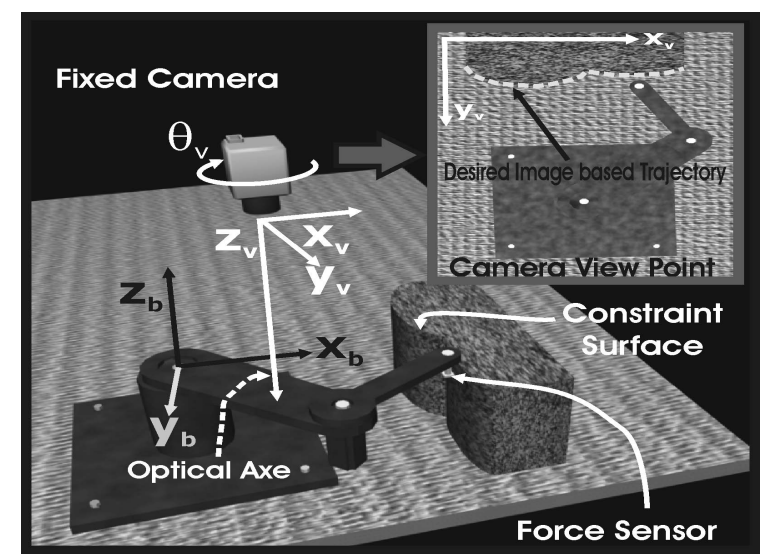

Fig. 1. Servoing of the visual servoing contact task.

\section{A. Visual force servoing}

Hybrid vision/force control approaches have been reported [2] [3], and none of them shows robustness to uncertainties on robot and camera parameters. In a different path, Hosoda et. al. [4] presents an adaptive robot controller to achieve contact tasks in an unknown environment, but the authors assumed that the movement of the camera-manipulator system is slow, considering the mapping from joint space to image space as constant. This assumption limits the dynamic system, and requires the exact knowledge of the robot kinematics, which is a very restrictive condition. Along similar developments Xiao et. al. [5] presents an interesting scheme for uncalibrated environment, but their approach does not deal

\footnotetext{
${ }^{1}$ Notice that the static state of the camera is not a generalized coordinate of the dynamical system, thus its slow latency is not an issue, and the system can be treated in the time domain.
}

with uncertainties of robot parameters, i.e., is an inverse dynamics position/force control scheme, which requires the exact knowledge of robot dynamics, and relies on a very complex control law. In the last year the authors [6], present an image-based control law under parametric uncertainties of robot and camera parameters, however the approach does not deals with uncertain restriction surface, next the work of [7] present an approach that deals with robot, camera and dynamic friction parameters only at simulation level, later in [8] the last approach is proved at experimental level, nevertheless, both approaches require the exact knowledge of the restriction surface. Finally, the authors [1] presents a scheme for visual servoing for constrained robots, and this scheme includes robot and camera uncertainties and deals with unknown contact surface, but only presents local convergence of visual tracking and force errors, this restricts the robot work-field to only achieve non-periodic tasks. The approach presented in this article uses decomposition of the joint space which, besides that produces global exponential convergence of tracking errors, also simplifies the proof, in comparision with [1] [6]. This is also one byproduct of this scheme.

\section{NONLINEAR ROBOT DYNAMICS}

\section{A. Constrained robot dynamics}

The constrained robot dynamics arises when its end-effector is in contact to infinitely rigid surface. Considering the generalized joint position $q \in \Re^{n}$ and joint velocity coordinates $\dot{q} \in \Re^{n}$, based on [9] and including friction dynamics, this system can be modeled by nonlinear differential algebraic equations as follows:

$$
\begin{aligned}
H(q) \ddot{q}+C(q, \dot{q}) \dot{q}+g(q) & =\tau+J_{\varphi+}^{T}(q) \lambda \\
\varphi(q) & =0
\end{aligned}
$$

where matrix $H(q) \in \Re^{n \times n}$ stands for the robot inertia matrix; $C(q, \dot{q}) \dot{q} \in \Re^{n}$ stands for the vector of centripetal and Coriolis torques; $g(q) \in \Re^{n}$ is the vector of gravitational torques, $J_{\varphi+}(q)=\frac{J_{\varphi}(q)}{J_{\varphi}(q) J_{\varphi}^{T}(q)}$ is the constrained normalized jacobian of the kinematic constraint $\varphi(q)=0$, or rigid surface with continuous gradient, and $\lambda$ is the constrained lagrangian, or contact force, and finally $\tau \in \Re^{n}$ stands for the vector of input torque control. An important property of robot dynamics, useful for stability analysis, is stated as follows:

Property I: Robot dynamics are linearly parameterizable in terms of a known regressor $y=y(q, \dot{q}, \dot{q}, \ddot{q}) \in \Re^{n \times p}$ and an unknown, but constant vector $\theta_{b} \in \Re^{p}$ of robot parameters as follows

$$
H(q) \ddot{q}+C(q, \dot{q}) \dot{q}+g(q)+J^{T}(q) B_{t} J(q) \dot{q}=y \theta_{b}
$$

Adding and subtracting the linear parametrization equation (3) to $(1)$, produces the open loop error equation

$H(q) \dot{S}_{q}=-C(q, \dot{q}) S_{q}-J^{T}(q) B_{t} J(q) S_{q}+\tau+J_{\varphi}^{T}(q) \lambda-y \theta_{b}$ with joint error surface $S_{q}$ is defined as

$$
S_{q}=\dot{q}-\dot{q}_{r}
$$

where $\dot{q}_{r}$ stands for the nominal reference of joint velocities. 


\section{CAMERA-ROBOT MODEL}

In order to design a proper nominal reference of joint velocities $\dot{q}_{r}$, the direct and inverse robot kinematics and the monocular static pin hole, with thin lens without aberration, camera model are used [10]. So, to this end, let robot direct kinematics be

$$
x_{b}=f(q)
$$

where $x_{b} \in \Re^{n}$ represents the position of robot end-effector in the robot work space and $f(\cdot): \Re^{n} \rightarrow \Re^{n}$. Then, the differential kinematics of robot manipulator is defined as follows

$$
\dot{x}_{b}=J(q) \dot{q}
$$

which relates velocities in cartesian space $\dot{x}_{b} \in \Re^{n}$ to joint space velocities $\dot{q} \in \Re^{n}$. The visual position $x_{v} \in \Re^{2}$ of robot end-effector in image space (screen) is given by [10],

$$
x_{v}=\alpha_{\lambda} R(\theta) x_{b}+\beta
$$

where $\alpha_{\lambda}$ is the scale factor ${ }^{2}$, and $R(\theta) \in S O(2)$ is the upper left $2 \times 2$ matrix of $R_{3}(\theta) \in S O(3) ; \beta \in \Re^{2}$, which depends on intrinsic and extrinsic camera parameters ${ }^{3}$. The subscript ' $v$ ' of $x_{v}$ denotes visual from visual space. In this way, the differential camera model becomes

$$
\dot{x}_{v}=\alpha_{\lambda} R(\theta) \dot{x}_{b}
$$

where $\dot{x}_{v} \in \Re^{2}$ determines the visual robot end-effector velocity, i.e. visual flow. Notice that the constant transformation $\alpha_{\lambda} R(\theta)$ maps statically robot cartesian velocities $\dot{x}_{b}$ into visual flow $\dot{x}_{v}$. Using (6), equation (8) becomes

$$
\dot{x}_{v}=\alpha_{\lambda} R(\theta) J(q) \dot{q}
$$

which relates visual flow $\dot{x}_{v}$ with joint velocity vector $\dot{q}$. Thus, the inverse differential kinematics of (9), in terms of visual velocities ${ }^{4}$, becomes

$$
\dot{q}=J_{\text {Rinv }} \dot{x}_{v}
$$

where $J_{R i n v}=J(q)^{-1} R(\theta)^{-1} \alpha_{\lambda}^{-1}$. This relation is useful to design the nominal reference of joint velocities $\dot{q}_{r}$ in the following section.

\section{Visual Orthogonalization Principle (VOP)}

Since the robot end-effector is in contact with the constrain surface, then

$$
\varphi(q)=0 \quad \forall t
$$

Taking the time derivative of (11), the OP arises

$$
\frac{d}{d t} \varphi(q)=0 \rightarrow \frac{\partial \varphi(q)}{\partial q} \frac{d q}{d t}=0 \Rightarrow \dot{\varphi}(q)=J_{\varphi}(q) \dot{q} \equiv 0
$$

\footnotetext{
${ }^{2}$ In this case, we are considering monocular camera model with nonredundant robot, thus $n \in \Re^{2}$ and $\alpha_{\lambda}$ will be considered as a scalar matrix $2 \times 2$.

${ }^{3}$ Focal distance, depth of field, translation of camera center to image center, distance between optical axe to the robot base, etc.

${ }^{4}$ With $J_{\text {Rinv }} \in \Re^{n \times n}$ whose entries are functions of robot and camera parameters.
}

This means that $J_{\varphi}(q)$ is orthogonal to $\dot{q}$. Clearly, an orthogonal projection of $J_{\varphi}(q)$ arises onto the tangent space at the contact point between the end-effector and the surface $\varphi(q)=0$, as proposed by [9]

$$
Q=I-J_{\varphi+}^{T}(q) J_{\varphi}(q)
$$

In other words, since the robot end-effector is in contact with the constraint surface, then

$$
Q \dot{q}=\dot{q} \quad \text { and } \quad Q Q \dot{q}=Q \dot{q} \equiv \dot{q}
$$

Substituting eq (10) in the last expression, an important property of the VOP arise:

$$
Q Q J_{R i n v} \dot{x}_{v}=Q J_{R i n v} \dot{x}_{v} \quad \text { and } \quad Q J_{R i n v} \dot{x}_{v}=J_{R i n v} \dot{x}_{v}
$$

\section{A. Global decomposition of joint space}

Considering a partition of the joint space $q$ as in [11]

$$
q=\left[q_{1}, q_{2}\right]^{T}
$$

where $q_{1} \in \Re^{m}$, and $q_{2} \in \Re^{n-m}$. Since the kinematics of the restriction $\varphi(q) \in \Re^{m}$, there are $m$ dependent states, which are defined in (13) as $q_{1}$. This partition is not arbitrary, thus to identify $q_{1}$ the jacobian of the restriction $J_{\varphi}(q)$ and the Gauss decomposition are used in order to define a non singular matrix $m \times m$ where the generalized coordinates of this matrix are indeed $q_{1}$. In accordance with the theorem of the implicit function, an open group $O \in \Re^{n-m}$ exists and a function $\Omega: \Re^{n-m} \rightarrow \Re^{m}$ such as:

$$
q_{1}=\Omega\left(q_{2}\right)
$$

locally exists, then $\varphi(q)=\varphi\left(\Omega\left(q_{2}\right), q_{2}\right)=0 \forall q_{2} \in O$. Now using the time derivative of (2) and its partitioning (13) leads to

$$
J_{\varphi}(q) \dot{q}=\left[J_{\varphi_{1}}(q) \dot{q}_{1}+J_{\varphi_{2}}(q) \dot{q}_{2}\right]=0
$$

where $J_{\varphi_{1}}(q) \dot{q}_{1} \in \Re^{m \times m}$ and $J_{\varphi_{2}}(q) \dot{q}_{2} \in \Re^{m \times n-m}$. Solving the above equation (15) for $\dot{q}_{1}$ rises

$$
\dot{q}_{1}=\Omega_{q_{2}} \dot{q}_{2} \quad \Omega_{q_{2}}=-\left[J_{\varphi_{1}}(q)\right]^{-1} J_{\varphi_{2}}(q)
$$

where $\Omega_{q_{2}}: \Re^{n-m} \rightarrow \Re^{m}$. Then joint velocities lies

$$
\begin{aligned}
\dot{q} & =Q \dot{q}_{2} \\
Q & =\left[\begin{array}{ll}
\Omega_{q_{2}} & I_{n-m}
\end{array}\right]^{T}
\end{aligned}
$$

with $Q \in \Re^{n \times(n-m)}$ with full column rank $\operatorname{rank}(n-m)$. 


\section{B. Visual-force error coordinated system.}

From the above implications and from (10), leads to

$$
\dot{q}_{=} Q J_{R i n v} \dot{x}_{v}
$$

These properties are fundamental to set the $V O P$, which allows to design $\dot{q}_{r}$ similarly to (18) and in terms of orthogonal nominal references of velocity $\dot{q}_{v}$ and force $\dot{q}_{f}$, as follows

$$
\dot{q}_{r}=\dot{q}_{v}+\dot{q}_{f}
$$

wherein $\dot{q}_{v}$ will depend on image-based coordinates and $\dot{q}_{f}$ will depend on integral of force coordinates. Naturally, from (18), equation (19) becomes

$$
\dot{q}_{r}=Q J_{R i n v} \dot{x}_{r}+\dot{q}_{f}
$$

Finally, and according to [9]

$$
\dot{q}_{r}=Q J_{R i n v} \dot{x}_{r}+\gamma_{F_{2}} J_{\varphi}^{T}(q) \dot{q}_{r f}
$$

for $\gamma_{F_{2}}>0$.

Let now the nominal visual reference of velocities be

$$
\begin{aligned}
\dot{x}_{r}= & \dot{x}_{v d}-\alpha \Delta x_{v}+S_{v d}-\gamma_{v_{1}} \int_{t_{0}}^{t} S_{v \delta}(\zeta) d \zeta \\
& -\gamma_{v_{2}} \int_{t_{0}}^{t} \operatorname{sign}\left(S_{v \delta}(\zeta)\right) d \zeta
\end{aligned}
$$

where $\dot{x}_{v d}$ stands for desired visual velocity trajectory, and $\Delta x_{v}=x_{v}-x_{v d}$ is the visual position error. Let the visual error surface be

$$
S_{v \delta}=\underbrace{\left(\Delta \dot{x}_{v}+\alpha \Delta x_{v}\right)}_{S_{v}}-\underbrace{S_{v}\left(t_{0}\right) e^{-\kappa_{v} t}}_{S_{v d}}
$$

where $\Delta \dot{x}_{v}=\dot{x}_{v}-\dot{x}_{v d}$ defines visual velocity error, $\kappa_{v}>0$ and $\gamma_{v_{i}}=\gamma_{v_{i}}^{T} \in \Re_{+}^{n \times n}, i=1,2$. Now, consider the following nominal force reference

$$
\begin{aligned}
\dot{q}_{r f}= & \Delta F-S_{F d}+\gamma_{F_{1}} \int_{t_{0}}^{t} S_{F \delta}(\zeta) d \zeta \\
& +\gamma_{F_{2}} \int_{t_{0}}^{t} \operatorname{sign}\left(S_{F \delta}(\zeta)\right) d \zeta
\end{aligned}
$$

for

$$
S_{F \delta}=\underbrace{\Delta F}_{S_{F}}-\underbrace{S_{F}\left(t_{0}\right) e^{-\kappa_{F} t}}_{S_{F d}}
$$

where

$$
\Delta F=\int_{t_{0}}^{t} \Delta \lambda(\zeta) d \zeta, \quad \Delta \lambda=\lambda-\lambda_{d}
$$

where $S_{F \delta}$ stands as the force error surface, with $\Delta \lambda$ as force tracking error and $\lambda_{d}$ as the desired contact force; $\kappa_{F}>0$, and $\gamma_{F_{i}}=\gamma_{F_{i}}^{T} \in \Re_{+}^{n \times n}, i=1,2$. Using equations (21), (22), (24) and (18) into (4), the joint error surface $S_{q}$ is

$$
\begin{aligned}
S_{q} & =Q J_{R i n v} \dot{x}_{v}-Q J_{R i n v} \dot{x}_{r}-\gamma_{F_{2}} J_{\varphi}^{T}(q) \dot{q}_{r f} \\
& =Q J_{R i n v} S_{v v}-\gamma_{F_{2}} J_{\varphi}^{T}(q) S_{v F}
\end{aligned}
$$

with

$$
\begin{aligned}
& S_{v v}=S_{v \delta}+\gamma_{v_{1}} \int_{t_{0}}^{t} S_{v \delta}(\zeta) d \zeta+\gamma_{v_{2}} \int_{t_{0}}^{t} \operatorname{sign}\left(S_{v \delta}(\zeta)\right) d \zeta \\
& S_{v F}=S_{F \delta}+\gamma_{F_{1}} \int_{t_{0}}^{t} S_{F \delta}(\zeta) d \zeta+\gamma_{F_{2}} \int_{t_{0}}^{t} \operatorname{sign}\left(S_{F \delta}(\zeta)\right) d \zeta
\end{aligned}
$$

where $S_{v v}$ stands for the extended visual manifold and $S_{v F}$ stands for the extended force manifold.

Remark 1. Notice that $S_{q}$ is composed of two orthogonal complements, $Q J_{\text {Rinv }} S_{v v}$ depending on image coordinate error, and $\gamma_{F_{2}} J_{\varphi}^{T}(q) S_{v F}$ depending on integral of contact force errors. Thus, tracking errors $\left(\Delta \dot{x}_{v}, \Delta x_{v}\right)$ and $\Delta F$ can be controlled independently with a single control loop, since they are mapped into orthogonal complements.

\section{UNCALIBRATED VOP SYSTEM}

The above definition assumes exact knowledge of $J_{\text {Rinv }}$. However, in practice, it stands as a very restricted assumption. Therefore, we need to design a uncertain manifold $\hat{S}_{q}$ taking into consideration the uncertainty of $J_{R i n v}$, and this involves that an estimation of $\varphi(q)$ must be used. To this end, consider

$$
\widehat{\dot{q}}_{r}=\widehat{Q} \widehat{J_{\text {Rinv }}} \dot{x}_{r}+\gamma_{F_{2}} \widehat{J_{\varphi}^{T}} \dot{q}_{r f}
$$

with $\widehat{J_{R i n v}}$ an estimated of $J_{R i n v}$, such that rank $\widehat{J^{-1}(q)}$ and $\widehat{R_{\alpha}^{-1}(\theta)}$ are full rank $\forall q \in \Omega$, where the robot workspace free of singularities is defined by $\Omega=$ $\left\{q \mid \operatorname{rank}(J(q))=n, \forall q \in \Re^{n}\right\}$, and $\forall \theta \in \Re$. The estimation of the contact surface is defined as $\widehat{\varphi(q)}$, which exhibits the next properties:

$$
\begin{aligned}
\widehat{\varphi(q)} & =e(q) \\
\frac{\widehat{d \varphi(q)}}{d t} & =\frac{\partial \widehat{\varphi(q)}}{\partial q} \frac{d q}{d t} \approx \dot{e}(q) \\
\widehat{J_{\varphi}(q)} \dot{q} & =\dot{e}(q)
\end{aligned}
$$

Where, $\widehat{J_{\varphi}(q)}$ is an estimation of $J_{\varphi}(q), e(q) \in C^{2}$ stands as an error of surface estimation, which is a function of the joint vector $q$, and $\dot{e}(q)$ is the time derivative of the error estimation, which preserves the next property $\|\dot{e}(q)\|<\zeta_{1}$, with $\zeta_{1} \in \Re_{+}$because $\varphi(q) \in C^{2}$. Accordingly

$$
\widehat{Q}=I-\widehat{J_{\varphi+}^{T}(q)} \widehat{J_{\varphi}(q)}
$$

where $\widehat{J_{\varphi+}^{T}(q)}=\frac{\widehat{J_{\varphi}^{T}(q)}}{\widehat{J_{\varphi}(q) \widehat{J_{\varphi}^{T}(q)}}}$. Then, $\widehat{Q}$ shows the next attributes

$$
\widehat{Q} \widehat{Q}=\widehat{Q}, \quad \widehat{Q} \dot{q} \neq \dot{q}, \quad \widehat{Q} \widehat{J_{\varphi+}^{T}(q)}=0
$$

Thus, substituting (29) into (4), we have the uncalibrated joint error surface

$$
\begin{aligned}
\hat{S}_{q} & =\dot{q}-\widehat{\dot{q}}_{r} \\
& =Q J_{R i n v} \dot{x}_{v}-\widehat{Q} \widehat{J_{R i n v}} \dot{x}_{r}-\gamma_{F_{2}} \widehat{J_{\varphi}^{T}} \dot{q}_{r f}
\end{aligned}
$$


where $\hat{S}_{q}$ is available because $\dot{q}$ and $\widehat{\dot{q}}_{r}$ are available. Adding and subtracting some terms to (32) we obtain:

$$
\begin{aligned}
\hat{S}_{q} & =Q J_{R i n v} S_{v v}-\gamma_{F_{2}} J_{\varphi}^{T} S_{v F}-\dot{q}_{\Delta r} \\
& =S_{q}-\dot{q}_{\Delta r}
\end{aligned}
$$

where

$$
\begin{aligned}
\dot{q}_{\Delta r} & =\Delta Q_{J} \dot{x}_{r}+\gamma_{F_{2}} \Delta J_{\varphi}^{T}(q) \dot{q}_{r F} \\
\Delta Q_{J} & =\widehat{Q} \widehat{J_{R i n v}}-Q J_{R i n v} \\
\Delta J_{\varphi}^{T}(q) & =\widehat{J_{\varphi}^{T}(q)}-J_{\varphi}^{T}(q)
\end{aligned}
$$

Notice that the uncalibrated $\hat{S}_{q}$ is in fact the calibrated $S_{q}$ plus a function that models the uncertainties. This parametrization is casted as an endogenous uncertainty and it is key in the following developments.

\section{OPEN LOOP ERROR EQUATION}

The parametrization of Property I can be written in terms of nominal references as follows

$$
H(q) \widehat{\ddot{q}}_{r}+C(q, \dot{q}) \widehat{\dot{q}}_{r}+g(q)=\hat{y}_{r} \theta_{b}
$$

Adding and subtracting (34) to (1), we obtain finally the following uncalibrated open loop error equation

$$
H(q) \widehat{\dot{S}_{q}}=\tau-C(q, \dot{q}) \widehat{S_{q}}+J_{\varphi+}^{T}(q) \lambda-\hat{y}_{r} \theta_{b}
$$

Now we are ready to present the main result.

\section{CONTROL DESIGN}

Assuming that $x_{v}, \dot{x}_{v}$ are measurable by the camera, $q, \dot{q}$ are measurable by encoder and tachometer respectively, as well as $\lambda, F$ by a force sensor, the task is designed such that desired image $\left(x_{v d}, \dot{x}_{v d}\right) \in \Omega_{x}$ and $\lambda \in C^{1}$, then we have the following.

Theorem 1 Assume that initial conditions and desired trajectories belong to $\Omega_{T}=\left[\Omega_{q}, \Omega_{x}\right]$, and consider the robot dynamics (1), (2) in closed loop with the following visual adaptive force-position control law

$$
\tau=-\left[K_{d}+K_{\Delta}|\lambda|\right] \widehat{S_{q}}+\widehat{J_{\varphi+}^{T(q)}}\left[\Delta F+\eta \int_{t_{0}}^{t} \operatorname{sgn}(\Delta F)\right]
$$

where $K_{d}, K_{\Delta} \in \Re_{+}^{2 \times 2}, \eta>0$. If $K_{d}$ is large enough and error of initial conditions are small enough, and if $\gamma_{v_{2}} \geq$ $\left.\left\|\frac{d}{d t}\left[Q_{\theta}^{\#}\left(\widehat{S_{q}}+\dot{q}_{\Delta r}\right)\right]\right\|, \gamma_{F_{2}} \geq \| \frac{d}{d t}\left[J_{\varphi}^{\#}\left(\widehat{S_{q}}+\dot{q}_{\Delta r}\right)\right]\right] \|$, then global exponential convergence of visual and force tracking errors is guaranteed.

Proof : The proof can be found in the appendix.

\section{EXPERIMENTAL STATION}

Robot parameters and constant gains used in the experiments are shown in Table I, and an image of the experimental setup is depicted in Fig. 2, meanwhile, camera parameters

\begin{tabular}{|c|c|c|c|c|c|}
\hline Par & Value & Gain & Value & Gain & Value \\
\hline$m_{1}$ & $7.1956 \mathrm{Kg}$ & $\overline{K_{d}}$ & $\operatorname{diag}(20,1.65)$ & $\overline{K_{\Delta}}$ & $\operatorname{diag}(5,2)$ \\
\hline$m_{2}$ & $1.8941 \mathrm{Kg}$ & $\gamma_{v_{1,2}}$ & $\operatorname{diag}(8,8)$ & $\gamma_{1}$ & $(3,3)$ \\
\hline$l_{1}$ & $0.4 \mathrm{~m}$ & $\alpha$ & $\operatorname{diag}(5,5)$ & $\left(B_{t}, \eta\right)$ & $(0.1,0.3)$ \\
\hline$l_{2}$ & $0.3 \mathrm{~m}$ & $\Gamma$ & 1 & $\kappa_{1}, \kappa_{2}$ & 20 \\
\hline
\end{tabular}
are: $\alpha_{\lambda}=\alpha_{s} \frac{\lambda_{v}}{\lambda_{v}-h}, \alpha_{s}=99500.0$ pixels $/ \mathrm{m}, h=1.6 \mathrm{~m}$, $\lambda_{v}=0.008, \beta_{s}=[-335,-218]$ pixels, $\theta=90^{\circ}$ (notice that this scheme does not presents any restriction for $\theta$, as other approaches [12], [13], etc.). The robot and camera parametric uncertainty is $10 \%$ for each parameter, i.e., $l_{1}, l_{2}, \alpha_{\lambda}$, etc.
TABLE I

PARAMETERS (Par) OF THE ROBOT, AND FEEDBACK GAINS.

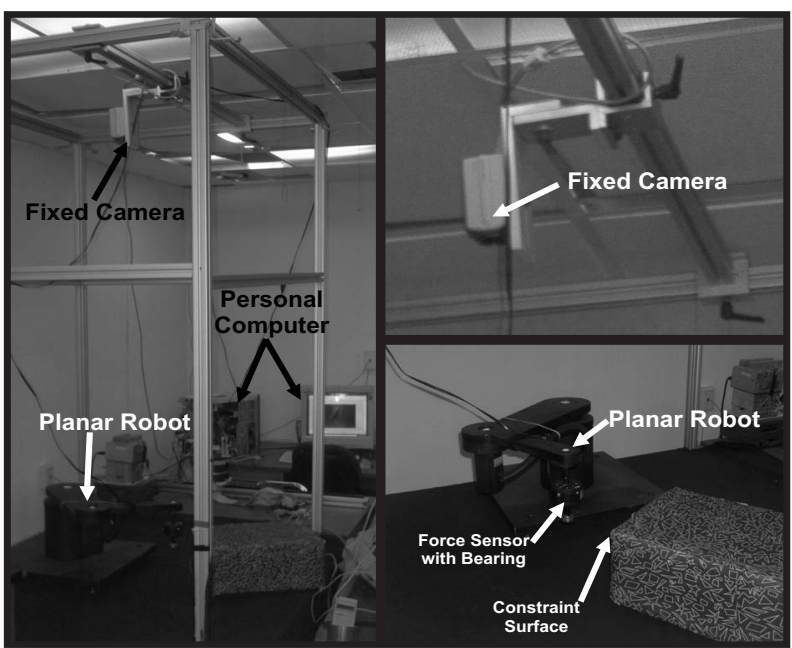

Fig. 2. Experimental setup.

\section{A. The hardware, software and firmware}

Fig. 3 shows the following experimental system configuration. Block $\mathbf{A}$ is composed by a $2.2 \mathrm{GHz}$ personal computer, running over Linux-RTAI OS. This, implements two realtime parallel processes. The first one, sets communication, at a sample rate of $30 \mathrm{~Hz}$, with the Block C, SONY VFWV500 camera via IEEE1394 protocol and controls the image processing in order to acquire the robot end-effector position in image space. The second process, Block $\mathbf{D}$, runs with a sampling rate of $1 \mathrm{KHz}$, reads the force sensor and encoders signals computing the torque output for Block B, where the direct-drive Yaskawa AC servomotors SGM-08A314 and SGM-04U3B4L with 2048 pulse encoders are directly coupled to the links of the 2-dof arm. The Block B is also integrated by two digital drives Yaskawa servopacks (SGD-08AS and SGDA-04AS), and a six-axis force-moment sensor 67M25AI40-200N12 by JR3 Inc., provided with a DSP Based Interface System for PCI bus. The force sensor is mounted to the end effector of the robot with a rigid aluminum probe with a bearing in its tip, as is shown in Fig.2. Communication between processes is done by real-time shared memory allocation. Low level programming in the Block A provides the interface to a Sensoray $626 \mathrm{I} / O$ card which contains internal quadrature encoder interface, with 14 bit analog resolution outputs and digital $I / O$. The user designs the desired image based trajectory, directly form the image displayed in the Block $\mathbf{A}$, and sets the desired force profile. Velocity is computed using a 


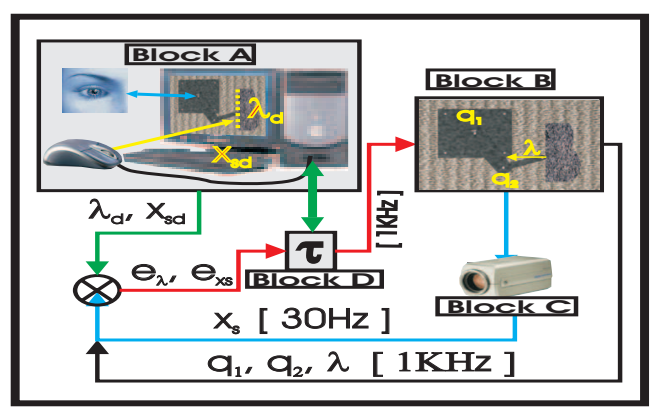

Fig. 3. Fast closed loop of dynamic state and slow static visual transformation.

dirty Euler numerical differentiation formula filtered with a lowpass second order Butterworth filter.

\section{B. The task}

The robot task is to move its tool-tip along a specified image-based trajectory over the steel surface while at the same time exerts a specified profile of force normal to the surface. The inertial frame of the whole system is at the base of the robot and the estimated contact surface is an $X Z$ plane located at $y=378$ pixel. The real surface exhibits a slope of $8.5^{\circ}$. The experiment is performed as follows

1) From $t=0 \mathrm{~s}$ to $t=2 \mathrm{~s}$. The end effector is requested to move, in free motion, i.e, $\widehat{J_{\varphi}^{T}}=0, \widehat{Q}=I^{5}$, from its initial condition until it makes contact with the surface. The end effector rests 1 seconds to establish stable contact.

2) From time $t=3 \mathrm{~s}$ to $t=6 \mathrm{~s}$. Once the tool-tip is in contact with the surface, the control force term is switched on and the tool-tip exerts a desired profile of force normal to the surface (0 to $7 \mathrm{~N}$ ) while moving along XY-plane from $(245,378)$ pixels to $(363,360)$ pixels.

3) From $t=6 \mathrm{~s}$ to $t=9 \mathrm{~s}$. The exerted force is incremented from 7 to 15 Newtons, while moving from $(363,360)$ pixels to $(265,375)$ pixels, as can be seen in Fig. 4 and Fig. 5 \& 6.

\section{Results}

First of all, notice that despite the nice piece of engineering of the JR3 force sensor, it delivers about $\pm 2 \mathrm{~N}$ of noise, and since desired contact force is very low, the noise can be observed in figures. A simple way to apparently get rid of this noise, is to set desired contact force $\lambda_{d}$ to a larger value, however such noise will be always present in there. Therefore, the small the desired contact force the more problematic the noise is. In the following figures we implement low desired contact force to test our scheme in more stringent conditions, however, as mentioned above, more better plots could be obtained simply by setting $\lambda_{d}$ to larger value.

\footnotetext{
${ }^{5}$ It is rather easy to prove that this scheme is stable for unconstrained motion.
}

We suppose that the estimate of the restriction surface can be described, in a very simple way as a normal plane depicted with the equation $y=378$ pixel, but the real restriction surface presents imperfections, as it shown in Fig. 6. However, as it can be seen in Fig. 4, The control law is capable to deal with the uncertainties in the equation of the restriction surface $\varphi(q)$, i.e. uncertainties in $J_{\varphi}(q), Q$. The seemingly high frequency is due this tasks requires very precise control, and due to the sensor resolution is limited to 1 pixel.

In Fig.4 the exerted force profile is shown, as can be seen, from $\left[\begin{array}{ll}0 & 2\end{array}\right]$ s the robot end effector performs free motion (near to $0 \mathrm{~N}$ ) until it makes contact with the surface (an overshoot in the contact force is presented due to contact transition), then it remains in that state 1 more seconds and from [3 6]s, increases the applied force smoothly from [0 7]N, while it moves forward (see Figs. $5 \&$ 6), and finally from [6 9]s once again, it increases the exerted force [ 715$] \mathrm{N}$, while it moves backward (see Figs. $5 \& 6$ ). The noisy signal is caused by the high precision of the JR3 sensor.

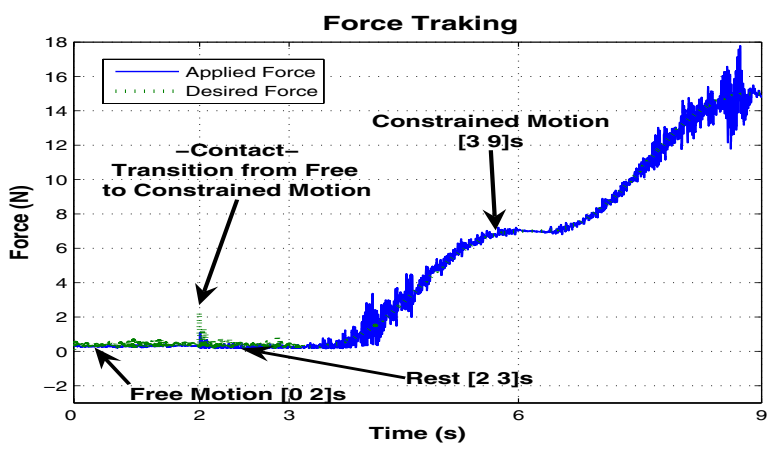

Fig. 4. Force Tracking

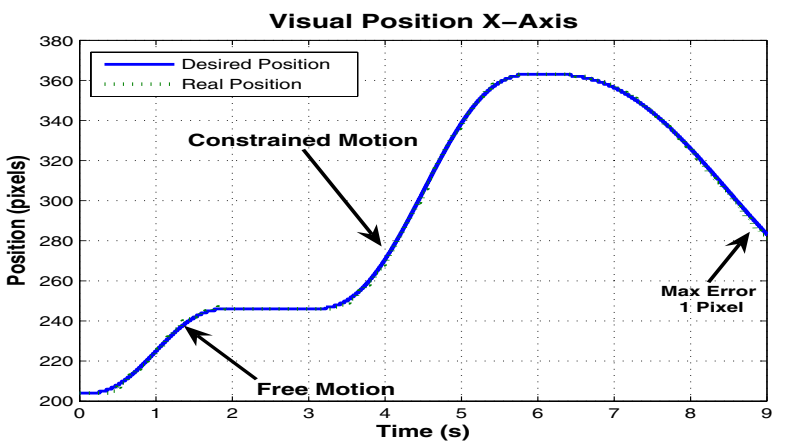

Fig. 5. End effector $\mathrm{x}$ position in image space (pixels).

\section{Conclusions}

This paper introduces a novel scheme for image based visual servoing for force control tasks in constrained dynamical robots and unknown environment. The main feature is the ability to fuse image coordinates into an orthogonal complement of joint velocities, and contact forces. This allows 


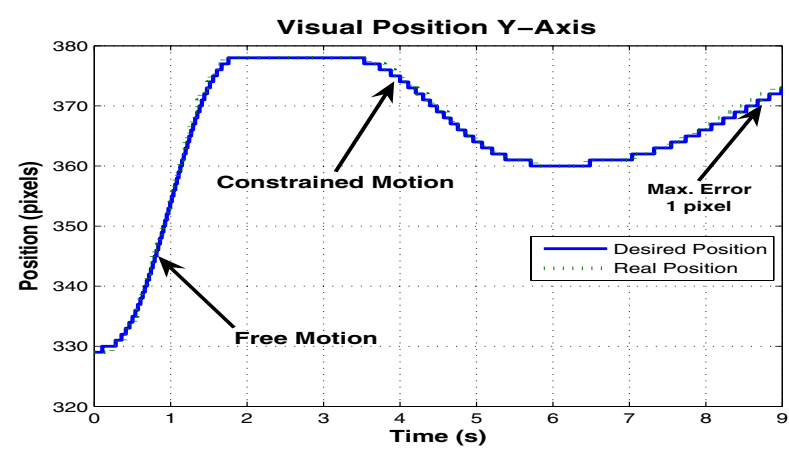

Fig. 6. End effector position in image space (pixels).

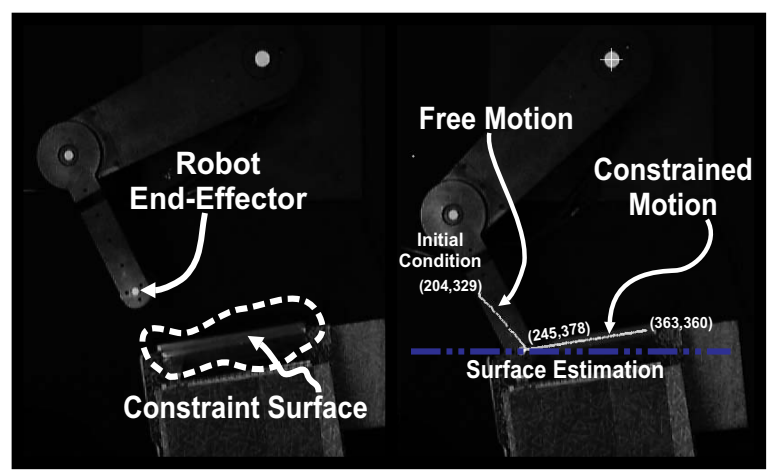

Fig. 7. Camera view point of the experimental validation.

to yield exponential convergence for image-based positionvelocity and contact forces even when robot parameters, camera parameters, analytical jacobian and restriction surface parameters are considered unknown. Notice that the stability is preserved even when the robot end-effector motion switches from free motion to constrained motion due to its passivity properties, under a set of conditions [14]. Experimental results comply with the theoretical properties.

\section{ACKNOWLEDGEMENTS}

E. Dean thanks CONACYT support under a doctoral scholarship \#158973. V. Parra-Vega acknowledges support from CONACYT project number 42797.

\section{REFERENCES}

[1] E.C. Dean-León, V. Parra-Vega, A. Espinosa-Romero, Visual Servoing for Constrained Robots in an Unknown Environment: A New Complete Theoretical Framework and its Experimental Validation, Proc. of the 36th Int. Symp. on Robotics. ISR 2005, Tokyo, Japan, WE321, p.p. 77, Nov-Dec, 2005.

[2] A. Namiki, Y. Nakabo, I. I. M. I., High Speed Grasping using Visual and Force Feedback. Proc.of the IEEE Int. Conf. on Robot. and Autom., Detroit, MI, 3195-3200, 1999.

[3] Garcia-Rodriguez, R. Dean-Leon, E. Parra-Vega, V. Ruiz-Sanchez, F., An adaptive neural network controller for visual tracking of constrained robot manipulators, Proc. of the 2005 American Control Conference, 2005., vol. 5, 3694 - 3700, June 2005.

[4] K. Hosoda, K. Igarashi, M. Asada, Adaptive Hybrid Control for Visual and Force Servoing in an Unknown Environment, IEEE Robot. and Autom. Magazine, 39-43, Dec-1998.
[5] Xiao, D., Sensor-hybrid Position/Force Control of a Robot Manipulator in an Uncalibrated Enviroment, IEEE Trans. Control Systm Technology, 8(4), 635-645, 2000.

[6] E.C. Dean-Leon, V. Parra-Vega, A. Espinosa-Romero, Image-based visual/force/position control under parametric uncertainties of camera and robot arm, 1st International Conference on Electrical and Electronics Engineering, 2004. (ICEEE), 321 - 326, June 2004.

[7] E.C. Dean-León, L.G. García, V. Parra-Vega, A. Espinosa-Romero, Uncalibrated Image-based Position-Force Adaptive Visual Servoing for Constrained Robots under Dynamic Friction Uncertainties, 2005 IEEE/RSJ International Conference on Intelligent Robots and Systems, 2005. (IROS 2005). 2013 - 2020, Aug. 2005.

[8] E.C. Dean-León, L.G. García, V. Parra-Vega, A. Espinosa-Romero, Visual servoing for constrained robots: a new complete theoretical framework and its experimental validation, Proc. of the 2005 IEEE/ASME International Conference on Advanced Intelligent Mechatronics, 1445 1452, 2005.

[9] S. Arimoto, Y. H. Liu and T. Naniwa, Model Based Adaptive Hybrid Control for Geometrically Constrained Robot Manipulator, Proc. IEEE Int. Conf. on Rob. and Autom., 618-623, 1993.

[10] S. Hutchinson, G. Hager, P. C., A Tutorial on Visual Servo Control, Trans. on Robot. and Autom., Vol. 6, 651-670, 1996.

[11] V. Parra, S. Arimoto, Y. H. Liuy T. Naniwa, Model-based adaptive hybrid control of robot manipulators under holonomic constraints, Proc. IFAC Symp.of Robot Control (SYROCO), 475-480,1994.

[12] Hsu, L., and Aquino, P., Adaptive Visual Tracking with Uncertain Manipulator Dynamics and Uncalibrated Camera, 1999 Proc. 38th IEEE Conference on Decision and Control, pp. 1248-1253.

[13] E. Zergeroglu, D.M. Dawson, M.S. de Queiroz, P. Setlur, Robust VisualServo control of Robot Manipulators in the Presence of Uncertainty, J of Robot Syst 20:(2), 93-106, 2003.

[14] P. Akella, V. Parra-Vega., Arimoto S. and T. Tanie, Discontinuous Adaptive Control for Robot Manipulators Executing Free and Constrained Tasks, Proc. of the IEEE Robotics and Automation, pages 3000-3007, San Diego (1994).

[15] V. Parra-Vega, S. Arimoto, Yun-Hui Liu, G. Hirzinger, P. Akella, Dynamic Sliding PID Control for Tracking of Robot Manipulators: Theory and Experiments, IEEE Trans. on Robot. and Autom., Vol. 19., 967-976, 2003.

[16] E.C. Dean-León, V. Parra-Vega, L.G. García-Valdovinos, A. EspinosaRomero, "Adaptive Visual Servoing for Constrained Robots under Jacobian, Joint Dynamic and Contact Viscous Friction Uncertainties", Procc. of the 16th IFAC WORLD CONGRESS, Prague, July 3-8, 2005.

\section{APPENDIX}

Proof of Theorem1: The closed loop dynamics between (36) and (35) yields

$$
\begin{aligned}
H(q) \widehat{\dot{S}_{q}} & =-C(q, \dot{q}) \widehat{S_{q}}+J_{\varphi+}^{T}(q) \lambda \\
& \left.-\hat{y}_{r} \theta_{b}-\left[K_{d}+K_{\Delta}|\lambda|\right] \widehat{S_{q}}+\widehat{J_{\varphi+}^{T(q}}\right) \eta S_{v F}
\end{aligned}
$$

The proof is organized in three parts.

Part I. Boundedness of Closed Loop Trajectories. Consider the following Lyapunov candidate function

$$
V=\frac{1}{2}\left[\hat{S}_{q}^{T} H(q) \hat{S}_{q}\right]
$$

which is a defined positive function (see eq.27, 28). Now, considering the time derivative of along the solutions of (37) as

$$
\begin{aligned}
\dot{V} & =-K_{d}\left\|\hat{S}_{q}\right\|_{2}^{2}+\left\|\hat{S}_{q}\right\| \psi-K_{\Delta}|\lambda|\left\|\hat{S}_{q}\right\|_{2}^{2} \\
& +{\widehat{S_{q}}}^{T} J_{\varphi+}^{T}(q) \lambda+{\widehat{S_{q}}}^{T} \widehat{J_{\varphi+}^{T}(q)} \eta S_{v F}
\end{aligned}
$$

where $\psi$ is a functional depending on the state and error manifolds, similarly to [15]. Substituting (30) and (32) in (39) 
lies

$$
\begin{aligned}
\dot{V} & \leq-K_{d}\left\|\hat{S}_{q}\right\|_{2}^{2}+\left\|\hat{S}_{q}\right\| \psi \\
& -K_{\Delta}|\lambda|\left\|\hat{S}_{q}\right\|_{2}^{2}+\widehat{S}_{q}^{T} J_{\varphi+}^{T}(q) \lambda \\
& -\gamma_{F_{2}} \eta\left\|S_{v F}\right\|_{2}^{2}+\dot{e}_{+}(q) \eta S_{v F}
\end{aligned}
$$

with $\dot{e}_{+}(q)=\frac{\dot{q}^{T} \widehat{J_{\varphi}^{T}(q)}}{J_{\varphi}(q) J_{\varphi}(q)^{T}} \equiv \frac{\dot{e}(q)}{J_{\varphi}(q) J_{\varphi}(q)^{T}}$.

If we consider the trivial solution $\dot{e}_{+}(q)=0$, which means two situations: a) $\Delta J_{\varphi}^{T}(q)=0$, i.e, there is no error in the restriction surface estimation or $b) \Delta J_{\varphi}^{T}(q)=\zeta_{2}, \zeta_{2} \in \Re$, this is, the estimation of the restriction surface presents a constant error. This consideration can be treated with approaches as [16], [7].

Now, considering $\left\|\dot{e}_{+}(q)\right\|<\zeta_{2}$, then (40) is

$$
\begin{aligned}
\dot{V} & \leq-K_{d}\left\|\hat{S}_{q}\right\|_{2}^{2}+\left\|\hat{S}_{q}\right\| \psi \\
& -K_{\Delta}|\lambda|\left\|\hat{S}_{q}\right\|_{2}^{2}+\widehat{S}_{q}^{T} J_{\varphi+}^{T}(q) \lambda \\
& -\gamma_{F_{2}} \eta\left\|S_{v F}\right\|_{2}^{2}+\zeta_{2} \eta S_{v F}
\end{aligned}
$$

Now if $K_{d}$ and are large enough, $K_{\Delta}>\left\|J_{\varphi+}^{T}(q)\right\|, \gamma_{F_{2}}>\zeta_{2}$ and the initial errors are small enough, we conclude the seminegative definiteness of (39) outside of hyperball $\varepsilon_{0}=$ $\left\{\widehat{S_{q}} \mid \dot{V} \leq 0\right\}$ centered at the origin, such as the following properties of the state of closed loop system arise

$$
\hat{S}_{q}, S_{v F} \in \mathcal{L}_{\infty} \rightarrow\left\|S_{v s}\right\|,\left\|S_{v F}\right\| \in \mathcal{L}_{\infty}
$$

Then, $\left(S_{s \delta}, \int \operatorname{sign}\left(S_{s \delta}\right)\right) \in \mathcal{L}_{\infty}$, and since desired trajectories are $C^{2}$ and feedback gains are bounded, we have that $\left(\widehat{\dot{q}}_{r}, \widehat{\ddot{q}}_{r}\right) \in \mathcal{L}_{\infty}$. The right hand side of (26),(33) shows that $\varepsilon_{1}>0$ exists such that $\left\|\widehat{\dot{S}_{q}}\right\| \leq \varepsilon_{1}$. This result shows global stability of $\widehat{S_{q}}$ and $\widehat{\dot{S}_{q}}$. Now we prove that the sliding modes arises.

Rewriting (33) in terms of two orthogonal vectors, we obtain

$$
\hat{S}_{q}=Q J_{\text {Rinv }} S_{v s}-\gamma_{F_{2}} J_{\varphi}^{T} S_{v F}-\dot{q}_{\Delta r}
$$

Since $\hat{S}_{q} \in \mathcal{L}_{2}$, and $J_{\text {Rinv }}, Q$ and $\dot{q}_{\Delta r}$ are bounded, then $Q J_{R i n v} S_{v s}$ is bounded and, due to $\widehat{\varphi(q)}$ is smooth and lies in the reachable robot space and $S_{v F} \rightarrow 0$, then $\gamma_{F_{2}} J_{\varphi}^{T} S_{v F} \rightarrow 0$. Now, taking into account that $\widehat{\dot{S}}_{q}$ is bounded, then $\frac{d}{d t}\left(J_{R i n v} Q S_{v s}\right)$ and $\frac{d}{d t}\left(\gamma_{F_{2}} J_{\varphi}^{T} S_{v F}\right)$ are bounded (this is possible because $\dot{J}_{\varphi}^{T}$ is bounded and so $\dot{Q}$ is). All this chains of conclusions proves that there exists constants $\varepsilon_{2}>0$ and $\varepsilon_{3}>0$ such that

$$
\left|\dot{S}_{v s}\right|<\varepsilon_{2},\left|\dot{S}_{v F}\right|<\varepsilon_{3}
$$

Now, we have to prove that for a proper selection of feedback gains $\gamma_{v_{1}}, \gamma_{v_{2}}$ and $\gamma_{F_{1}}, \gamma_{F_{2}}$ then trajectories of visual position and force converges to zero. This is possible if we can prove that sliding modes are established in the visual subspace $Q$ and in the force subspace $J_{\varphi}^{T}(q)$.

Part II: Second Order Sliding Modes.
Part II.a: Sliding modes for the velocity subspace. According to (26),(33), multiplying by $Q^{T}$ and substituting (27),(28) give rise to

$$
\left(Q^{T} Q\right)^{-1} Q^{T}\left\{S_{q}+\dot{q}_{\Delta r}\right\} \equiv J_{R i n v} S_{v v}
$$

If we multiply (44) by $R_{\alpha}(\theta) J(q)$, we have

$$
Q_{\theta}^{\#}\left\{S_{q}+\dot{q}_{\Delta r}\right\}=S_{v \delta}+\gamma_{v_{1}} \int_{t_{0}}^{t} S_{v \delta}+\gamma_{v_{2}} \int_{t_{0}}^{t} \operatorname{sign}\left(S_{v \delta}\right)
$$

with $Q_{\theta}^{\#}=R_{\alpha}(\theta) J(q)\left(Q^{T} Q\right)^{-1} Q^{T}$.

Taking the time derivative of (45), and multiply it by $S_{v \delta}^{T}$, it produces

$$
S_{v \delta}^{T} \dot{S}_{v \delta} \leq-\mu_{v}\left|S_{v \delta}\right|-\gamma_{v_{1}}\left\|S_{v \delta}\right\|^{2}
$$

where $\mu_{v}=\gamma_{v_{2}}-\varepsilon_{4}$, and $\varepsilon_{4} \geq\left|\frac{d}{d t}\left[Q_{\theta}^{\#}\left\{S_{q}+\dot{q}_{\Delta r}\right\}\right]\right|$. Thus, we obtain the sliding condition if $\gamma_{v_{2}}>\varepsilon_{4}$, such as $\mu_{v}>0$ of (46) guarantees the sliding mode at $S_{v \delta}=0$ at $t_{v}=\frac{\left|S_{v \delta}\left(t_{0}\right)\right|}{\mu_{v}}$. However, notice that for any initial condition $S_{v \delta}\left(t_{0}\right) \stackrel{\mu_{v}}{=} 0$, then $t_{v}=0$, which implies that the sliding mode at $S_{v \delta}(t)=0$ is guaranteed for all time. Part II.b: Sliding modes for the force subspace. In the same way as the sliding mode for visual subspace equation (26),(33) leads to

$J_{\varphi}^{\#}(q)\left\{S_{q}+\dot{q}_{\Delta r}\right\}=S_{F \delta}+\gamma_{F_{1}} \int_{t_{0}}^{t} S_{F \delta}+\gamma_{F_{2}} \int_{t_{0}}^{t} \operatorname{sign}\left(S_{F \delta}\right)$

where $J_{\varphi}^{\#}(q)=\left(-\gamma_{F_{2}} J_{\varphi} J_{\varphi}^{T}(q)\right)^{-1} J_{\varphi}$. Taking time derivative of (47) and multiply it by $S_{F \delta}^{T}$, (47) becomes

$$
S_{F \delta}^{T} \dot{S}_{F \delta} \leq-\mu_{F}\left|S_{F \delta}\right|-\gamma_{F_{1}}\left\|S_{F \delta}\right\|^{2}
$$

where $\mu_{F}=\gamma_{F_{2}}-\varepsilon_{5}$, and $\varepsilon_{5} \geq \frac{d}{d t}\left[J_{\varphi}^{\#}(q)\left\{S_{q}+\dot{q}_{\Delta r}\right\}\right]$. If $\gamma_{F_{2}}>\varepsilon_{5}$, then a sliding mode at $S_{F \delta}(t)=0$ is induced at $t_{f} \leq \frac{\left|S_{F \delta}\left(t_{0}\right)\right|}{\mu_{F}}$, but $S_{F \delta}\left(t_{0}\right)=0$, thus $S_{F \delta}\left(t_{0}\right)=0$ is guaranteed for all time.

Part III: Exponential convergence of tracking errors.

Part III.a: Visual tracking errors. Since a sliding mode exists for all time at $S_{v \delta}(t)=0$, then we have

$$
S_{v}=S_{v d} \forall t \rightarrow \Delta \dot{x}_{v}=-\alpha \Delta x_{v}+S_{v}\left(t_{0}\right) e^{-\kappa_{v} t}
$$

this implies that the visual tracking errors globally tends to zero exponentially fast, this is $x_{v} \rightarrow x_{v d}, \dot{x}_{v} \rightarrow \dot{x}_{v d}$, implying that the robot end-effector converges to the desired image $x_{v d}$, with given velocity $\dot{x}_{v d}$.

Part III.b: Force tracking errors. Since a sliding mode at $S_{F \delta}(t)=0$ is induced for all time, this means $\Delta F=$ $\Delta F\left(t_{0}\right) e^{-\kappa_{F} t}$. Moreover, in [15] it is shown that the convergence of force tracking errors arises, thus $\lambda \rightarrow \lambda_{d}$ exponentially fast. QED.

Remark 2. The control scheme is based on the Lyapunov stability theory along with the variable structure control theory for second order sliding modes, which presents robustness for a class of bounded unmodeled dynamics with exponential and global stability and continuous structure in contrast to first order sliding modes theory. 